\title{
Preface to Variational Analysis for Beginners
}

\author{
Annamaria Barbagallo ${ }^{1} \cdot \operatorname{Radu}_{B^{\prime} t^{2}} \cdot$ Claudia Sagastizábal $^{3} \cdot$ Michel Théra $^{4,5}$
}

Accepted: 8 October 2020/ Published online: 17 October 2020

(C) Springer Nature B.V. 2020

The journal dedicates this issue, entitled "Variational Analysis for Beginners", to readers who value knowledge and wish to learn or remember theoretical developments and technical tools that are specific to this exciting area.

The volume presents a collection of educational and self-contained works that were written to make more accessible and widespread among practitioners the topic under consideration. To highlight the power and usefulness of Variational Analysis for people not familiar with the domain, all important concepts are accompanied by illustrative examples.

This issue is a follow-up of the 71st Workshop on "Advances in Nonsmooth Analysis and Optimization" (NAO19) that was held within the framework of the International School of Mathematics "Guido Stampacchia" at the "Ettore Majorana" Foundation and Centre for Scientific Culture, Erice (TR), Sicily, from June 24 to July 1, 2019. The aim of the Workshop was to review and discuss recent developments of the theory of Nonsmooth Analysis and Optimization, and to provide a forum for fruitful interaction in closely related areas. Nonsmooth problems appear in many fields of applications, such as data mining, image denoising, energy management, optimal control, neural network training, economics and computational chemistry and physics. Motivated by these applications Nonsmooth Analysis had a considerable impulse that allowed the development of sophisticated methodologies for solving challenging related problems.

The contents of this special issue, authored by invited speakers of NAO19, can be summarized as follows.

In A Discussion of Probability Functions and Constraints from a Variational Perspective Wim van Ackooij introduces the topic of probability constraints, a popular modelling mechanism in applications. The work clarifies, adopting a Variational Analysis point of view,

Claudia Sagastizábal sagastiz@unicamp.br

1 Department of Mathematics and Applications "R. Caccioppoli”, University of Naples Federico II, Via Cintia - Complesso Universitario Monte S.Angelo, 80126 Naples, Italy

2 Faculty of Mathematics, University of Vienna, Oskar-Morgenstern-Platz 1, 1090 Vienna, Austria

3 IMECC - UNICAMP, Rua Sergio Buarque de Holanda, 651, Campinas, SP 13083-859, Brazil

4 XLIM UMR-CNRS 7252, Université de Limoges, Limoges, France

5 Federation University Australia, Ballarat, Australia 
several issues regarding theoretical properties and algorithms, highlighting open research avenues whenever possible.

In Continuous Dynamics Related to Monotone Inclusions and Non-smooth Optimization Problems, Ernö Robert Csetnek presents the main important techniques and tools from Variational Analysis used for first and second order continuous time approaches for monotone inclusions and nonsmooth optimization problems.

In A Discussion on Variational Analysis in Derivative-Free Optimization, Warren Hare shows how many successful Derivative-Free Optimization algorithms rely heavily on tools and results from Variational Analysis.

In Nonsmoothness in Machine Learning: Specific Structure, Proximal Identification, and Applications, Franck Iutzeler and Jérôme Malick focus on exploiting, from a Variational Analysis perspective, the specific structure of nonsmooth optimization problems appearing in machine learning.

In The $A B C$ of $D C$ Programming, Welington de Oliveira presents a short tutorial on difference-of-convex optimization, surveying and highlighting some important facts about DC functions, optimality conditions, and recent algorithms.

In the tutorial Set-Convergence and Its Applications, Johannes O. Royset reviews the central concept of set-convergence and explain its role in defining a notion of proximity between sets, especially for epigraphs of functions and graphs of set-valued mappings. The development leads to an approximation theory for optimization problems and generalized equations with profound consequences for the construction of algorithms. Examples illustrate the importance of set-convergence in stability analysis, error analysis, construction of algorithms, statistical estimation, and probability theory.

We are very grateful to the authors and referees for their valuable contributions and careful work.

Annamaria Barbagallo

Radu Boț

Claudia Sagastizábal

Michel Théra

Publisher's Note Springer Nature remains neutral with regard to jurisdictional claims in published maps and institutional affiliations. 